Internist 2009 · 50:1189-1190

DOI 10.1007/s00108-009-2521-9

Online publiziert: 17. Oktober 2009

๑) Springer Medizin Verlag 2009

\author{
R. Kolloch ${ }^{1} \cdot$ M. Grond ${ }^{2}$ \\ ${ }^{1}$ Klinik für Allgemeine Innere Medizin, Nephrologie und \\ Diabetologie, Evangelisches Krankenhaus Bielefeld \\ ${ }^{2}$ Klinik für Neurologie, Kreisklinikum Siegen
}

\section{Schlaganfall als Manifestation erhöhten kardiovaskulären Risikos}

\author{
Eine interdisziplinäre Herausforderung
}

Der Schlaganfall stellt in den westlichen Ländern mittlerweile die häufigste Gefäßerkrankung dar und rangiert damit noch vor dem Herzinfarkt und der peripheren arteriellen Verschlusskrankheit. Am Beispiel von Hessen konnte kürzlich gezeigt werden, dass sich aufgrund der demographischen Entwicklung die Zahl der Schlaganfallpatienten bis zum Jahr 2050 fast verdoppeln wird, d. h. in Hessen wird sich die Zahl der jährlichen Schlaganfallpatienten von ca. 21.00o auf über 35.0oo erhöhen. Die überwiegende Zahl der Patienten verstirbt an dieser Krankheit nicht, ein Großteil überlebt diese Erkrankung mehr oder weniger stark behindert.

Der Schlaganfall ist damit die häufigste Ursache für bleibende Behinderungen in unserer Gesellschaft. Die Folgen eines Schlaganfalls stellen nicht nur eine erhebliche Belastung für den Patienten und die Angehörigen dar, sondern auch eine erhebliche Belastung für das Sozialsystem. So ist davon auszugehen, dass im Durchschnitt für jeden Schlaganfallpatienten lebenslange direkte Kosten von über $50.000 €$ entstehen. Dies würde bis zum Jahr 2025 eine finanziellen Belastung von fast $110 \mathrm{Mrd}$. $€$ bedeuten. Diese Belastung stellt eine der größten Herausforderungen für das Gesundheits- und Sozialsystem dar.

Jeder 4. Schlaganfallpatient erleidet in den nächstens 5 Jahren ein weiteres schweres Gefäßereignis wie erneuten Schlaganfall, Myokardinfarkt oder einen vaskulären Tod. Der Schlaganfallpatient stellt damit einen vaskulären Hochrisikopatienten und damit eine interdisziplinäre Herausforderung für Neurologen und insbesondere Internisten und Hausärzte dar.

Neuere Entwicklungen, insbesondere in der Akuttherapie, vermögen mittlerweile effektiv den Anteil behinderter Patienten nach Schlaganfall zu reduzieren. Hier sind insbesondere die Thrombolysetherapie sowie die interdisziplinäre StrokeUnit-Behandlung zu nennen. Die intravenöse Thrombolysetherapie kann aufgrund neuester Studien bis 4 1/2 h nach Symptombeginn effektiv und sicher eingesetzt werden.

\section{( Die TIA ist gleichbedeutend mit einem ischämischen Schlaganfall und hat Notfallcharakter}

Die Verlängerung des bisherigen Zeitfensters um 90 min ist in der Praxis sehr relevant, da viele Schlaganfallpatienten erst zwischen der 2. bis 4. h nach Symptombeginn in der Klinik eintreffen. Die Verlängerung des therapeutischen Fensters darf aber auf keinen Fall dazu führen, dass sich der Behandlungsablauf in der Klinik unter der Vorstellung verzögert, dass der Zeitdruck jetzt kleiner ist. Mit jeder Minute, mit der die Lyse früher begonnen wird, steigt deren Effektivität.

Während sich in der Akuttherapie der Slogan "time is brain" mittlerweile etabliert hat, spielt auch in der Sekundärprophylaxe die Zeit eine immer größere Rolle. Der Beginn einer Sekundärprophylaxe muss frühzeitig nach dem ischämischen Ereignis begonnen werden. So konnte gezeigt werden, dass durch die sofortige Einleitung einer sekundärprophylaktischen Behandlung nach transitorisch ischämischer Attacke (TIA) im Vergleich zu einem Behandlungsbeginn nach im Durchschnitt 21 Tagen die Zahl der Schlaganfälle in den nächsten 3 Monaten um $80 \%$ reduziert werden kann. Insgesamt muss auch der Begriff der TIA neu überdacht werden. Der Begriff ist historisch und es liegt die Annahme zugrunde, dass Symptome, die sich innerhalb von $24 \mathrm{~h}$ zurückbilden, keinen morphologischen Schaden hinterlassen. Durch den vermehrten Einsatz der Schlaganfall-Magnetresonanztomographie konnte gezeigt werden, dass selbst bei einer Symptomdauer unter einer Stunde bei jedem 3. Patienten eine morphologische Schädigung gefunden werden kann. Die TIA ist somit kein „Schlägelchen“, sondern gleichbedeutend mit einem ischämischen Schlaganfall und hat Notfallcharakter.

Auch die Bewertung der Karotisstenose befindet sich in der Diskussion, auch hier gibt es einen Zeitfaktor: Während bisher 
streng zwischen einer symptomatischen und einer asymptomatischen Stenose unterschieden wurde, setzt sich zunehmend die Sichtweise einer Krankheitsentität durch. Die Karotisstenose ist als Marker einer systemischen, progredienten und in vielen Fällen aggressiven Arteriosklerose zu betrachten, die mit einem deutlich erhöhten vaskulären und hierbei insbesondere kardiovaskulären mehr noch als zerebrovaskulären Risiko einhergeht. Daher ist ein Patient mit einer Karotisstenose während der in der Regel langen asymptomatischen Phasen (residente Phase) als vaskulär hochgradig gefährdeter $\mathrm{Pa}$ tient leitliniengerecht und zielwertorientiert gefäßprophylaktisch zu behandeln. Nur während der in der Regel kurzen aktiven Phasen, in denen eine fokal neurologische Symptomatik auftritt, stellt sich zeitlich sehr befristet die Frage nach einer Intervention und hier nach der aktuellen Datenlage bevorzugt der Operation. Diese Intervention sollte aber idealerweise in einem Zeitfenster von 2-4 Wochen nach dem ischämischen Ereignis durchgeführt werden, danach ist die Effektivität fraglich.

Die Gestaltung einer adäquaten Sekundärprophylaxe ist sehr stark durch die Definition und Abklärung der auslösenden Faktoren geprägt. Die Aufdeckung und Therapie seltenerer Ursachen des Schlaganfalls, wie zum Beispiel entzündliche Gefäßerkrankungen, erfordern eine enge, interdisziplinäre Zusammenarbeit. Kardioembolische Ursachen mit klarer Indikationsstellung für eine dauerhafte orale Antikoagulation, insbesondere beim Vorhofflimmern, stellen in der alltäglichen Umsetzung ein großes Problem dar. Nur ca. 40\% der Patienten mit einer eindeutigen Indikation für den Einsatz von Marcumar werden entsprechend behandelt. Gerade in dieser Hinsicht ist die enge Zusammenarbeit von Neurologen und Internisten gefordert. Erste Ergebnisse beim Einsatz der neu entwickelten direkten oralen Thrombininhibitoren mit vereinfachter Handhabung der Therapiekontrolle lassen hoffen, dass die Ergebnisse der Langzeitantikoagulation entscheidend verbessert werden könnten.

Die Förderung einer engen und vernetzten Kooperation aller beteiligten Disziplinen wird es ermöglichen, bei der Prä- vention, Diagnostik, Akuttherapie und Sekundärprävention des Schlaganfalls bedeutsame Verbesserungen zu erzielen. Mit der vorliegenden Ausgabe von „Der Internist" hoffen wir, dazu einen Beitrag leisten zu können.

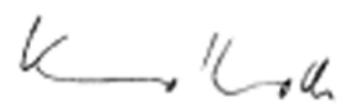

Prof. Dr. R. Kolloch

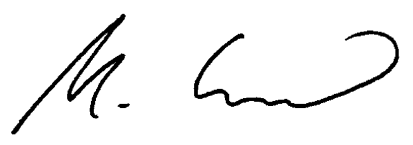

Prof. Dr. M. Grond

\section{Korrespondenzadresse}

Prof. Dr. R. Kolloch

Klinik für Allgemeine Innere Medizin, Nephrologie und Diabetologie, Evangelisches Krankenhaus Bielefeld Burgsteig 13, 33617 Bielefeld

rainer.kolloch@evkb.de

\section{Neuer Marker für die Blutgefäßbildung in Tumoren}

Wissenschaftler haben ein Protein als möglichen Marker für die Blutgefäßbildung in Tumoren identifiziert. Damit Tumoren wachsen können, müssen sie an die Blutversorgung angeschlossen werden. Dazu ist ein Tumor zur Angiogenese in der Lage. Ein beim Tumorwachstum beteiligtes, als EDB bezeichnetes Protein, ist in gesundem Gewebe praktisch nicht nachweisbar, kommt aber in Tumorgewebe in großer Menge vor. Da es sich an Stellen konzentriert, wo neue Blutgefäße wachsen, eignet sich EDB als Marker für Angiogenese. Wissenschaftler haben nun einen Antikörper gegen bestimmte EDB-Formen entwickelt, der mit normalem Gewebe nicht reagiert, jedoch hochspezifisch mit Tumorgewebe. Damit steht den Forschern nun eine Methode zur Verfügung, mit der sie die Neubildung von Blutgefäßen in Tumorzellen nachweisen können.

Literatur: Balza E, Sassi F, Ventura E et al (2009) A novel human fibronectin cryptic sequence unmasked by the insertion of the angiogenesis-associated extra type III domain B. Int J Cancer 125:751-758

Quelle: Deutsches Krebsforschungszentrum (DKFZ), www.dkfz.de 